\title{
Techniques for Comparing Four-Terminal-Pair Admittance Standards
}

\author{
R. D. Cutkosky \\ Institute for Basic Standards, National Bureau of Standards, Washington, D.C. 20234
}

(June 26, 1970)

\begin{abstract}
Some of the advantages of four-pair admittance standards and some of the special problems encountered in their measurement are pointed out. Detailed descriptions of three distinct types of fourpair bridges and some of their limitations are presented. These three bridges form a vital part of a very precise absolute measurement of resistance based on a calculable capacitor being undertaken at the National Bureau of Standards, but are believed to be of more general usefulness.
\end{abstract}

Key words: AC direct-reading ratio set; ac measurements; bridge; coaxial chokes; defining transformers; equal power bridge; four-pair standards; frequency-dependent bridge; quadrature bridge.

\section{Introduction}

The quest for improved accuracy in the measurement of audiofrequency admittances has led from the now universally accepted practice of utilizing two pair standards, such as so-called three-terminal capacitors for high-impedance devices and such as mutual inductors for low-impedance devices, to a composite system combining the advantages of both techniques. A theoretical justification of this composite system in which the standards are provided with four pairs of terminals was published in 1964 [1] ${ }^{1}$, but with the exception of a paper by Homan [2] which was limited to measurements on fairly small impedances, no systematic investigation of four-pair admittance measurements has yet been described. The process of converting to fourpair measurement systems has been underway at the National Bureau of Standards for several years, during which time many of the special problems involved in their use have been investigated. This paper is an attempt to describe some of the techniques which have evolved, and to indicate the order of precision that may be obtained.

The techniques described in this paper are of general applicability but were developed for the specific purpose of comparing the reactances of capacitors having nominal values of $10 \mathrm{pF}$ with the resistance of a special $1000-\Omega$ resistor having a negligible frequency dependence [3]. The three basic types of bridges used for making this comparison are described here in some detail. It is intended to calibrate the $10-\mathrm{pF}$ capacitors by means of a calculable capacitor now under construction at NBS. A de comparison of the 1000- $\Omega$ resistor with the bank of $1-\Omega$ resistors presently main-

' Figures in brackets indicate the literature references at the end of this paper. taining the NBS unit of resistance will then complete an absolute measurement of the ohım.

Because of the large number of intervening steps between the calculable capacitor and the bank of $1-\Omega$ resistors, very elaborate precautions are necessary to maintain a high level of accuracy. Using the techniques outlined here, it is expected that the principal uncertainties in the measurement sequence will be due to mechanical imperfections in the calculable capacitor.

Although a rather complex sequence of auxiliary balances is required with the bridges described in this paper, they are not prohibitively time-consuming for the limited number of comparisons needed in an absolute ohm determination. These techniques would be considerably less attractive for a bridge in constant use, but this would be less of a drawback in a computeroperated system. It is considered that techniques similar to those described in this paper are essential if uncertainties must be kept smaller than 1 part in $10^{8}$ over a large range of impedances at audiofrequencies.

The equivalent circuit of a four-pair admittance standard is shown in figure la, taken from the paper cited above [1], and in an abbreviated form, in figure 1b. The standard may be viewed as a device in which the ratio of current in pair 3 to the open circuit voltage at pair 2, subject to the condition that both the voltage and current at pair 4 are zero, is the parameter of interest. This parameter has the dimensions of admittance and may be written

$$
P_{32}^{14}=\frac{i_{3}}{e_{2}} \mid i_{2}=i_{4}=0, \quad e_{4}=0 .
$$

It may be shown using figure la that

$$
P_{32}^{14}=Y_{t}\left(1+Y_{2} Z_{2}\right)\left(1+Y_{3} Z_{3}\right) \text {. }
$$


An interesting reciprocity theorem also exists, which shows that $P_{32}^{14}=P_{23}^{41}$ for any four-pair network.

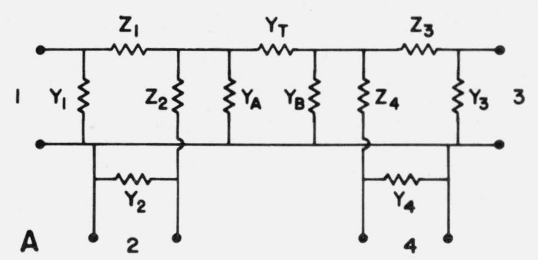

B

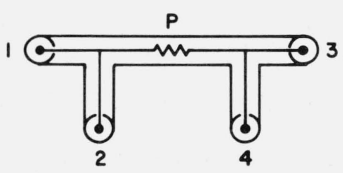

FIGURE 1. Four-pair admittance standards: (a) Complete circuit. (b) Pictorial representation.

Some properties of four-pair admittance standards which make them particularly useful are their relative insensitivities to variations in series impedances and shunt admittances in the leads as is implicit in (2), and the fact that standards of either very small or very large admittance may be constructed in such a way that their four-pair admittances are nearly identical with their ordinary two-pair admittances obtained by either open circuiting or short circuiting the two extra terminal pairs. These properties are discussed in the reference cited above [1].

Ordinarily the relationship between the four-pair admittance of a standard and its admittance when treated as a two-pair standard is of interest only when the standard is some kind of absolute or calculable instrument, and it is necessary to determine the difference between the calculated admittance at some internal and inaccessible location, and the effective four-pair admittance at the external connection points. Examples of this type of standard are calculable capacitors, calculable inductors, and resistors having calculable phase angles or ac-dc differences. Such devices are outside the scope of this paper, which is restricted to the problem of comparing four-pair admittance standards with each other.

Implicit in the adoption of either a four-pair or a two-pair approach is the requirement that the connections are indeed treated as pairs, and that there is no net current from one terminal pair to another. Many advantages result from grouping the terminals of a standard in pairs. Consider the simple case of a threeterminal capacitor provided with two coaxial connectors and with a permanent connection between the shields of these connectors. Conceptually the measurement of the capacitor consists of applying a voltage, $e$, to one connector pair and measuring the short circuit current $i$ at the other connector pair. The transfer admittance of the device is then given by

$$
Y=j \omega C+G=\frac{i}{e} .
$$

The transfer admittance defined in this way is a function of the impedance in the connection between the shields of the coaxial connectors, but there is no ambiguity in the definition as there would be if a single ground connection of unspecified placement were used for both the input and output.

One of the principal reasons for defining the standards in terms of isolated coaxial connector pairs as sketched above is that under these conditions there is exactly the same current but with reversed sign in the inner wire of each cable as there is in the surrounding coaxial shield, and therefore the magnetic field exterior to both cables is zero. This eliminates possible mutual inductive couplings between the cables used to interconnect a number of components together to form a bridge. Likewise, even if stray magnetic fields were present in the vicinity of the coaxial cables, no induced voltages would be produced by these fields to affect the measurement. Although these arguments pertain only to the connecting leads of the devices, the devices themselves can be quite easily isolated from each other by providing them with individual mu-metal shields when this is needed.

Four-pair admittance standards are constructed with four coaxial connectors, and the advantages gained by preventing net currents from flowing between the four connector pairs are very great, as this eliminates external magnetic fields and their effects on the measurement. In this respect, the practice of dealing with five terminal standards having a single, common ground connection cannot be recommended.

It is necessary to maintain the conditions of zero net currents in the cables of components defined as outlined above when they are interconnected to form a bridge circuit. Coaxial chokes were developed for dealing with this problem [4], and are effective in attenuating the net currents in the cables by a factor of several hundred. Unfortunately the net currents are not completely suppressed by coaxial chokes. A method for estimating the errors caused by incomplete suppression of net currents by coaxial chokes is described later in this paper.

Most of the bridge circuits appearing in this paper show explicitly the coaxial nature of the components. This was done to emphasize the importance of considering the return currents in the shields. After some practice in dealing with circuits drawn in this way, they are much easier to relate to the physical bridge setup than are the conventional textbook bridge circuits. Some difficulty may be experienced at first in translating the still useful and important body of classical bridge theory to the coaxial form of presentation. It may be an aid in understanding some of the circuits in this paper to redraw them with a common ground point. If this is done, the similarities between many of the auxiliary balance systems used here and common classical techniques, such as yoke and lead balancing in Kelvin double bridges, and the use of Wagner and conjugate Wagner arms in ac bridges, can be readily seen. Redrawing the circuits in this way must be thought of purely as an aid to understand- 
ing, because any attempt actually to construct a circuit without proper attention to the details of the shield return circuits would result in large and erratic errors dependent upon the accidental arrangement of the leads.

A final but important introductory point about comparisons of four-pair admittances, or indeed any other kind of admittances, is that even though the standards are defined subject to certain constraints on the currents and voltages, it is not necessary when comparing two such standards to satisfy any of the defining conditions; it is merely required that the same result be obtained for the ratio of the four-pair admittances being compared as would have been obtained if the conditions had all been satisfied. This observation opens up a very wide range of possibilities in the design of bridge circuits, and is related to the techniques described by Thompson for dealing with bridges involving multiple balances [4]. The Kelvin bridge for comparing fourterminal de resistors also makes use of this principle, since currents exist in the potential leads of the resistors, but cause no errors.

\section{Basic Comparison Circuits}

Figure 2 shows four four-pair admittances connected in a bridge circuit involving six generators and six detectors. The balance procedure consists (for example) of arbitrarily fixing one generator, and then adjusting the other five generators and one of the four

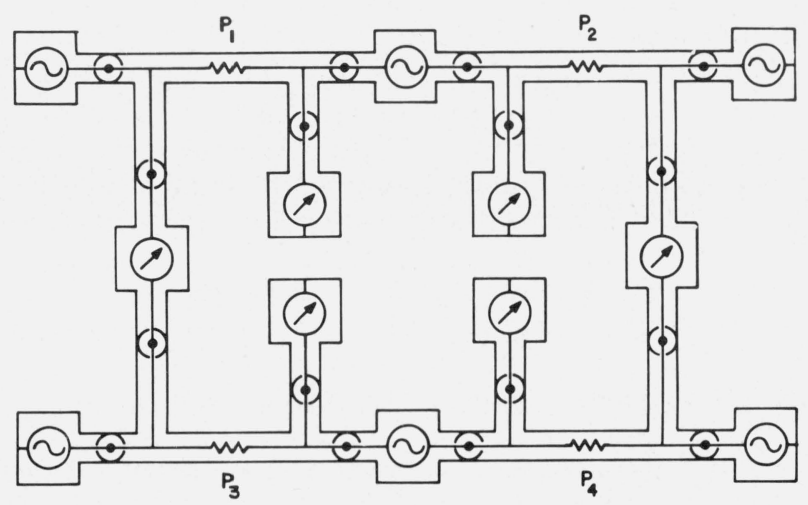

Figure 2. Elementary four-pair bridge.

four-pair admittances until all six detectors are balanced. The balance condition is $P_{1} P_{4}=P_{2} P_{3}$. If all generators and detectors are interchanged, the bridge network retains its form, and it may be shown, using the reciprocity theorem for four-pair networks, that the balance condition is unchanged.

The scheme of figure 2 contains a number of drawbacks, chief among which is the difficulty in simultaneously nulling six detectors, all of which are affected in various ways by the six adjustable parameters. This problem is solved in principle by mixing the various null detector responses in a suitable combining network to obtain six new null detector responses, each of which is a weighted average of the six original null detector inputs. The combining network parameters are chosen so that the matrix relating the new detector responses to the adjustable parameters is diagonal $[4,5]$. In this case, only the detector which responds to the adjustable four-pair admittance, called the main detector, is of interest, and the others need to be balanced only if the non-diagonal elements of the matrix are not exactly zero.

A more fundamental problem encountered in realizing figure 2 has to do with shunt admittances to ground associated with the detectors which compare the open circuit voltage of one standard with that of another, and with shunt admittances to ground associated with the generators between the current leads of two adjacent standards. By the reciprocity theorem the effects of these sets of leads are similar, and any solution found for one problem may be immediately applied to the other. The nature of the problem and the order of magnitude of the possible errors likely to occur may be seen from (2), which shows that the effect of admittance to ground in the potential lead is to load the leads joining the internal junction points of the standard with the point at which the open circuit potential is to be measured. The effect of admittance to ground at the current lead is to shunt some of the current to ground before it enters the adjacent standard.

The lead effects described above are unlikely to exceed a few parts in $10^{7}$ at audiofrequencies, unless interconnecting cables longer than several meters are used. Even with very long cables, no errors result provided that these cables are treated as part of the standard, so that their effects are the same when the standard is calibrated as when it is used for calibrating another standard. However, the stabilities of the series impedances and shunt admittances of the cables must be considered.

The network used to interconnect the ends of the two cables and the null detector or generator is in principle a three-pair network, since the interaction of the null detector or generator terminal pair must be included in the matrix description of the network. This network must be carefully constructed so that the admittances shunting the cables from the standards are small, equal, and measurable; and so that the interaction of the null detector or generator with the other two terminal pairs is simple to analyze. An ideal threepair circuit for accomplishing the desired objectives would provide a direct, completely reproducible connection between the two coaxial cables, except for an infinitesimal gap between their inner conductors, and with provision for connecting a null detector or generator across this gap. A network electrically equivalent to the above is employed at NBS which we call a defining transformer, shown pictorially in figure 3. A particular advantage of this network is that one side of the detector or generator terminal pair is at ground potential.

When used in the potential leads of the bridge shown in figure 2, the potential leads from the two standards are connected to terminals $A$ and $B$ of the defining transformer. The direct internal connection between the inner conductors, in connection with the 
return through the outer shield, provides a one-turn loop around the high-permeability core shown shaded. A detector is connected to a 100 -turn winding on this core via the coaxial connector $C$, and indicates a null

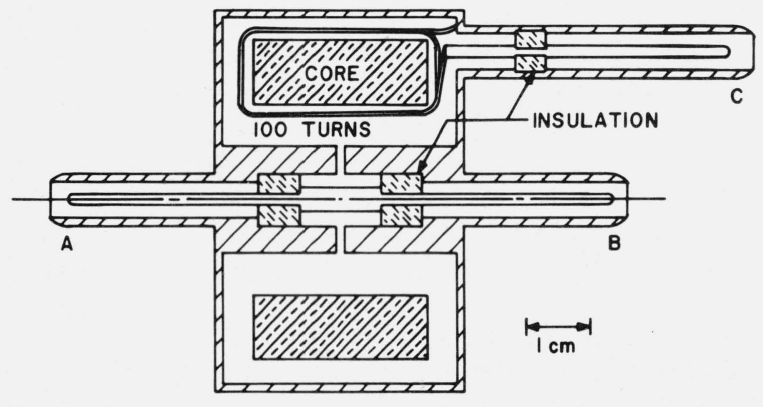

Figure 3. "Defining transformer" for terminating current and potential leads of four-pair standards.

if the potentials at $A$ and $B$ are equal. Capacitance between the 100-turn winding and the connection between the inner connectors of $A$ and $B$ could cause substantial errors. An internal shield with a thin air gap as shown eliminates this problem. Incomplete shielding can be detected by interchanging $A$ and $B$.

When using defining transformers in a bridge, the potential leads are considered to terminate at the center of the device, in the region of the insulated gap in the inner shield. A number of nearly identical defining transformers have been built such that they may be used interchangeably to terminate the potential leads of the standards without changing the loads on the leads. These devices are used in the current leads also, in which case a generator may be substituted for the null detector.

Some objection may be made to the use of these devices, in that the standard is no longer an entity in itself, but has a definition dependent upon a termination; which might more properly be considered as part of the measuring instrument. Precedents do exist for this type of procedure, an example being the use of a precision coaxial connector on a two-terminal capacitor, for which a correct measurement requires a mating precision connector. In practice a very substantial simplification of our measurements was found to result from the introduction of defining transformers.

The technique which we use for measuring the effects of net currents between one terminal pair and another in a bridge containing coaxial chokes is rather cumbersome, but provides a reliable measure of the errors caused by non-ideal coaxial chokes. It is first assumed that every ground loop in the system to be investigated contains a coaxial choke, and that the minimum possible number of chokes to accomplish this purpose is used. This does not imply that one cannot add as many ground loops as may be desired, but simply that in the final circuit all ground loops are interrupted with a minimum number of coaxial chokes. Singling out a particular choke for investigation, a single turn of wire is wrapped around the core of the choke and connected to an auxiliary detector. The deflection of this detector is a measure of the voltage tending to drive current through the choke.

A quantitative measure of this voltage may be obtained using the voltage insertion transformer shown in figure 4. This device consists of a high permeability

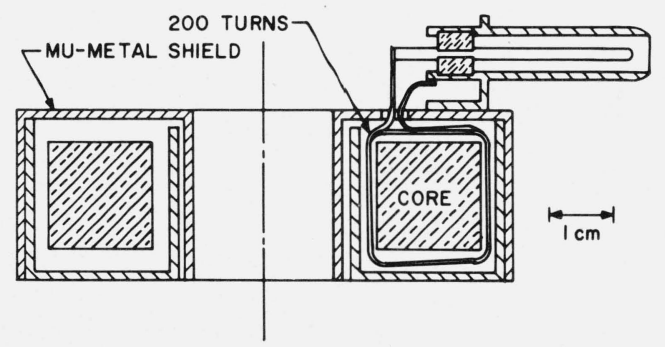

FIGURE 4. Voltage insertion transformer for investigating errors caused by net currents in coaxial cables.

toroidal core wrapped with 200 turns of wire connected to a coaxial connector. A toroidal mu-metal shield surrounds the core and winding, so that negligible magnetic fields exist outside of the shield when the 200 -turn winding is excited. In use, the cable leading to the choke selected for testing is passed through the hole in the shield of this device, forming a 200:1 transformer. A voltage source derived from the same oscillator supplying the bridge, and adjustable in both real and imaginary components, is connected to the 200 -turn winding. We find that an operational amplifier circuit described in an earlier paper [6] is convenient for this purpose.

The voltage source is adjusted until the auxiliary detector monitoring the voltage across the coaxial choke registers a null. The real and imaginary parts of the adjustable voltage required to obtain this null are recorded. The next step is to remove the auxiliary detector and to change the adjustable voltage by an amount large enough to yield sufficient sensitivity but not large enough to drive the choke into a nonlinear region. The real and imaginary parts of the change in balance point of the bridge caused by this change in applied voltage are recorded. A straightforward manipulation of the various complex quantities obtained by this scheme allows one to calculate the real and imaginary parts of the error caused by incomplete suppression of net current by the coaxial choke.

All of the coaxial chokes in the system are evaluated in this way. An algebraic addition of the individual errors then gives the total error, which is applied as a correction to the raw results of the measurement.

The technique sketched above could be generalized for a system with $M$ chokes by providing each of the $M$ chokes with voltage insertion transformers, and by bringing all of the choke voltages simultaneously to a null as indicated by $M$ auxiliary detectors. In this case no net currents with their attendant errors would exist, and there would be no corrections to apply to the bridge balance. The complete system applied in the circuit of figure 2 could then be viewed as one with 
$M+6$ adjustable parameters and with $M+6$ null detectors. One could conceivably construct a combining network to diagonalize the matrix relating these two vector quantities to make the effects of net choke currents less important. This has not yet been required for our purposes, since the residual errors due to net currents through the chokes are small enough to be measured with adequate precision using the extrapolation technique sketched above.

It is very important that one uses the minimum number of chokes necessary to break all ground loops when the normal loop voltages are measured by balancing them out as indicated above. If two chokes of equal impedance were in series in a particular loop, the method described would assign the total loop voltage to each choke, when in reality each choke would have across it only half of the total voltage. A simple test for multiply choked loops is to measure the voltages at each choke produced by exciting each loop in turn with a voltage insertion transformer. If the nondiagonal terms of the resultant matrix are much smaller than the diagonal terms, no multiply choked loops exist.

It is found in measuring the effects of the chokes in a given system that they can often be placed in categories according to the amount of voltage driving the loop, and according to the effect of a loop voltage on the bridge balance. The measurement error caused by a coaxial choke having insufficient impedance is large only if the loop voltage driving a choke is large and if a voltage in this loop has a strong effect on the bridge balance. It is usually possible to arrange a bridge in such a way that no choke has both undesirable characteristics. This usually requires the addition of extra ground connections with an extra choke for each added connection. The procedure for accomplishing this is at this time largely empirical.

Coaxial chokes do not provide the only means for suppressing net currents in coaxial cables. Isolation transformers are used in some cases, and net currents can then result from unbalanced interwinding capacitances. If the interwinding capacitances are very poorly balanced, the effective ground loop voltage can be quite high, and the accurate measurement of the errors due to the resultant ground loop current is rather difficult. In such cases it is usually best to measure the current directly, for example by converting the voltage insertion transformer to a current sensor by connecting a phase-sensitive voltmeter in parallel with a low impedance load to the 200-turn winding.

About the only other errors likely to occur in a fourpair bridge are those caused by stray couplings between the various components. These effects can usually be made negligible with electrostatic and electromagnetic shields, and tests for the effectiveness of these shields are not difficult to perform. Eccentric coaxial cables usually cause no serious problems, but must be considered. In some cases acoustic couplings can be troublesome, particularly if the generator and detector circuits both contain partially magnetized transformers. The 100:1 bridge described in section 5 suffered from this effect initially, but after the cores were carefully demagnetized and after the addition of some acoustic insulation, the errors due to acoustic coupling were reduced to a few parts in $10^{10}$. The mechanism responsible for the acoustic coupling involves magnetostriction in the core of the transformer in the generator circuit. If the core is partially magnetized, the acoustic output of the core will contain a component at the same frequency as the generator in addition to the expected but less troublesome components at the even harmonics of the generator frequency. The resultant mechanical excitation of the transformer in the detector circuit causes no trouble unless the detector transformer is also partially magnetized, in which case a fundamental frequency component of current can be generated.

Some care must be taken when adjusting the meters of the null detector to zero deflection in the absence of a signal. It had been the practice at NBS to disconnect the admittance standards of a bridge from the voltage transformer to which they were normally connected and to zero the meters with the voltage transformer excited. This technique eliminates most of the errors due to magnetic coupling from the oscillator and voltage transformer to the detector, and also eliminates some of the errors caused by imperfect coaxial chokes. When the choke errors are measured independently as sketched above, this procedure could result in a double correction for some of the choke errors.

\section{Quadrature Bridge}

As the first example of an actual four-pair bridge, we will use a type of frequency-dependent bridge of which two-pair versions have been in existence for some time $[4,7,8,9]$. The circuit is basically the same as that described by Thompson [4] and uses the twintee detector coupling network proposed by him. The NBS bridge contains two $10^{5}-\Omega$ four-pair resistors and two $1-n F$ four-pair capacitors, and operates at an angular frequency of $10^{4} \mathrm{rad} / \mathrm{s}$, which corresponds to a frequency of about $1592 \mathrm{~Hz}$. Figure 5 shows a partial schematic of the NBS four-pair quadrature bridge, in which $D_{1}$ through $D_{8}$ are null detectors, $T_{5}$ through $T_{9}$ are defining transformers, and $P_{1}$ through $P_{4}$ are the four-pair admittance standards. Seven of the eight complex adjustments required to balance the eight null detectors are indicated by either admittances or generators with arrows through their symbols. These seven adjustments are required only for realizing the defining conditions for the four-pair admittances, and may be uncalibrated. The eighth complex adjustment required to balance the bridge is made through the use of two seven-dial inductive voltage dividers $T_{2}$ and $T_{3}$, which drive small fixed capacitors labeled $Y_{5}$ and $Y_{6}$. This complex pair of adjustments serves to indicate the relationship between $P_{1}, P_{2}, P_{3}$, and $P_{4}$. Using the notation indicated on figure 5 , we have when all detectors are balanced

and

$$
i_{1}=e_{a} P_{1}+e_{d} Y_{5} \approx-e_{c} P_{2}
$$

$$
i_{2}=e_{b} P_{4}+e_{f} Y_{6} \approx-e_{c} P_{3} \text {. }
$$




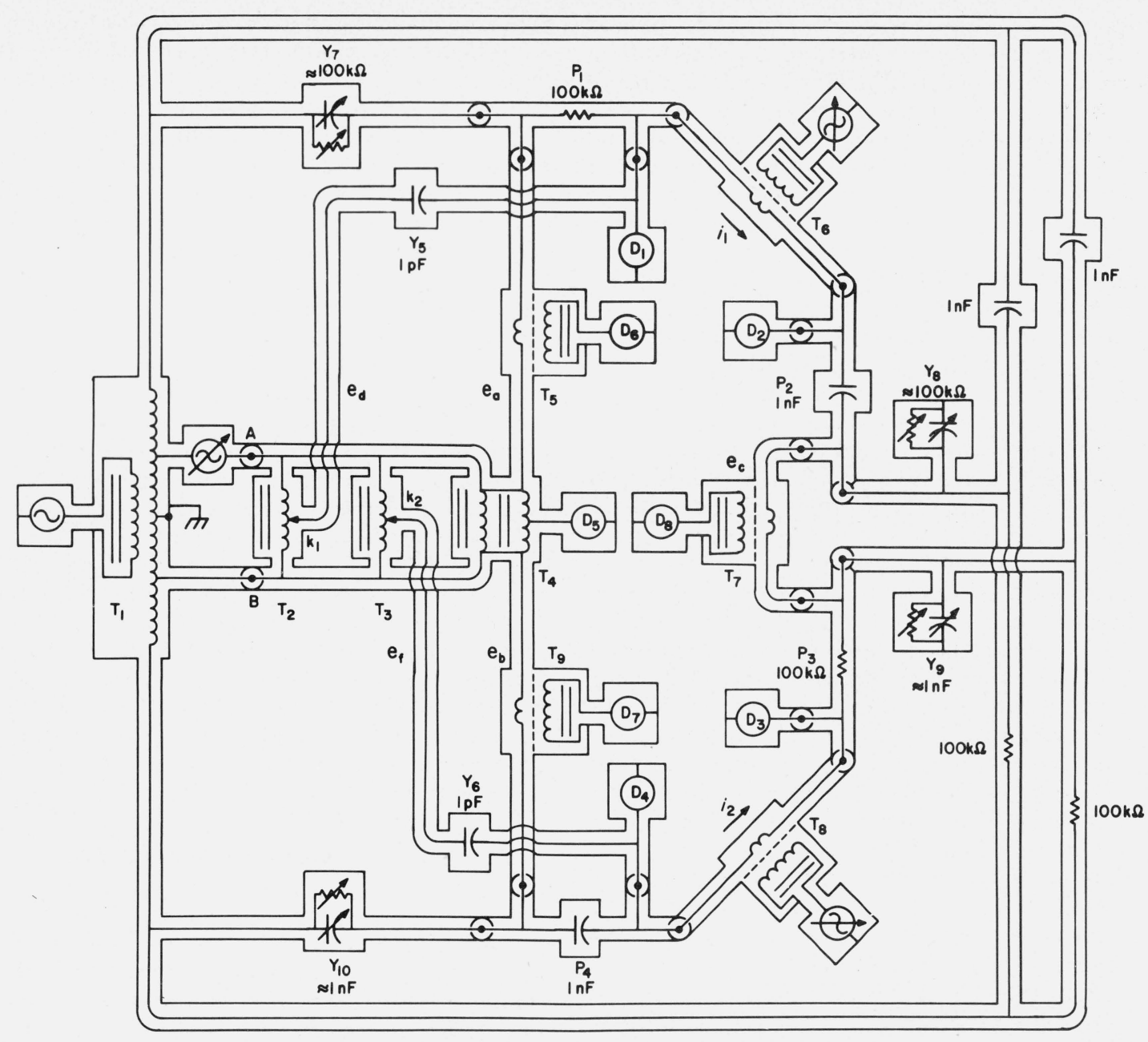

FIGURE 5. Four-pair quadrature bridge with detector combining network deleted.

If we represent the ratio of $T_{4}$ by $e_{b}=-e_{a}(1+\delta)$ with $\delta$ complex and $|\delta| \ll 1$, and since $e_{d} \approx e_{a}\left(2 k_{1}-1\right)$ and $e_{f} \approx-e_{b}\left(2 k_{2}-1\right)$, we have the balance condition $P_{3}\left[P_{1}+Y_{5}\left(2 k_{1}-1\right)\right] \approx-P_{2}(1+\delta)\left[P_{4}-Y_{6}\left(2 k_{2}-1\right)\right]$.

A repeat measurement is made with the connections interchanged at points $A$ and $B$, and with the connections between $T_{4}$ and the defining transformers $T_{5}$ and $T_{9}$ interchanged. This reverses the sign of $\delta$ to first order, and also reverses $T_{2}$ and $T_{3}$, so that

$$
e_{d}^{\prime} \approx e_{a}\left(1-2 k_{1}^{\prime}\right) \quad \text { and } \quad e_{f}^{\prime} \approx-e_{b}\left(1-2 k_{2}^{\prime}\right) .
$$

The resultant balance condition is

$$
P_{3}\left[P_{1}+Y_{5}\left(1-2 k_{1}^{\prime}\right)\right] \approx-P_{2}(1-\delta)\left[P_{4}-Y_{6}\left(1-2 k_{2}^{\prime}\right)\right] \text {. }
$$

Averaging (3) and (4) we have

$P_{3}\left[P_{1}+Y_{5}\left(k_{1}-k_{1}^{\prime}\right)\right] \approx-P_{2}\left[P_{4}-Y_{6}\left(k_{2}-k_{2}^{\prime}\right)\right]$.
If we let $P_{n}=G_{n}+j \omega C_{n}, Y_{5}=j \omega C_{5}$ and $Y_{6}=j \omega C_{6}$ and assume that $G_{1} \approx G_{3} \approx \omega C_{2} \approx \omega C_{4}$ and that $\omega C_{1} \ll G_{1}$, $\omega C_{3} \ll G_{3}, G_{2} \ll \omega C_{2}$ and $G_{4} \ll \omega C_{4}$, we may separate real and imaginary parts of (5) to obtain

$$
\frac{G_{3} G_{1}}{\omega^{2} C_{2} C_{4}} \approx 1+\frac{C_{6}}{C_{4}}\left(k_{2}-k_{2}^{\prime}\right)
$$

which relates the main components of the four-pair admittances, and

$$
\frac{\omega C_{1}}{G_{1}}+\frac{\omega C_{3}}{G_{3}}+\frac{G_{2}}{\omega C_{2}}+\frac{G_{4}}{\omega C_{4}} \approx \frac{G_{5}}{C_{2}}\left(k_{1}^{\prime}-k_{1}\right)
$$

which relates the phase angles of the four-pair admittances.

The approximations made in deriving (6) and (7) cause no errors exceeding 2 parts in $10^{10}$ for the NBS four-pair quadrature bridge. Under less ideal conditions, the second-order correction terms might be required. 
The nominally $1: 1$ transformer $T_{4}$ in figure 5 is of two-stage construction $[6,10]$, with a mu-metal shield between the first and second stages, and with another mu-metal shield surrounding the entire transformer. It can be seen that when the null detectors $D_{6}$ and $D_{7}$ are balanced, the output terminal pairs of $T_{4}$ are subject to open circuit conditions as defined at the central reference planes of the defining transformers $T_{5}$ and $T_{9}$. The voltages $\mathrm{e}_{a}$ and $e_{b}$ in the equations above are referred to these reference planes in a manner analogous to the definitions of the open circuit voltages of the four pair standards. Reversal of $T_{4}$ is accomplished by reconnecting the cables at the points of entry to $T_{5}$ and $T_{9}$, which has a negligible effect on the open circuit ratio.

Three of the eight null detectors shown in figure 5 are not operated on by the combining network used with the bridge. The detectors labeled $D_{6}, D_{7}$, and $D_{8}$ connected to $T_{5}, T_{7}$, and $T_{9}$ are brought to a null one at a time by adjusting $Y_{7}, Y_{10}$, and either $Y_{8}$ or $Y_{9}$, after which these detector terminals are shorted to reduce the effect on the main detector of a slight error in these auxiliary balances. This technique works very well for adjusting $Y_{7}$ and $Y_{10}$, but some convergence problems exist with the balance of $Y_{8}$ or $Y_{9}$. Ideally, one would like to null the detector $D_{8}$ connected to $T_{7}$ by adjusting $Y_{8}-Y_{9}$ while keeping $Y_{8}+Y_{9}$ fixed. One of the other balance conditions turns out to involve $Y_{8}+Y_{9}$ but not $Y_{8}-Y_{9}$. In practice, we have been able to achieve fast convergences of these two auxiliary balances without the elaboration of ganged switches by manually tracking $Y_{8}$ with $Y_{9}$.

The guiding principle behind the combining network used with the bridge (see fig. 6) is to combine the various detector terminal pairs two at a time and further to combine the new detector terminals so formed until only one detector terminal pair remains. We begin by combining the detector terminals $D_{1}$ and $D_{2}$ of $P_{1}$ and $P_{2}$ to form a single detector terminal $D_{9}$ which does not respond to excitation of $T_{6}$, and by similarly combining the detector terminals $D_{3}$ and $D_{4}$ of $P_{3}$ and $P_{4}$, to form a single detector terminal $D_{10}$ which does not respond to excitation of $T_{8}$. The initial combining network linking the detector terminals $D_{1}$ and $D_{2}$ can be understood by letting $e_{a}=e_{c}=0$ in figure 5 and observing that the circuit between $T_{6}$ and $D_{9}$ in figure 6 is a Schering bridge, for which a complex balance may be obtained by adjusting the two variable capacitors $C_{11}$ and $C_{12}$. The circuit is adjusted by actually connecting the bridge generator to the input lead of $T_{6}$ rather than to the input of $T_{1}$. After $C_{11}$ and $C_{12}$ are adjusted, the input terminal of $T_{6}$ is shorted. The result is that although the voltage at neither $D_{1}$ nor $D_{2}$ is exactly nulled, these voltages are very small; and since changing the excitation on $T_{6}$ does not change the voltage at $D_{9}$, no error results from failure to adjust this excitation to obtain individual nulls on the detector pairs $D_{1}$ and $D_{2}$. The combining network between the detector terminals of $D_{3}$ and $D_{4}$ is identical with that described above, and is adjusted by varying $C_{13}$ and $C_{14}$. The relative sizes of the admittances are chosen to produce a minimal effect on the bridge sensitivity and on the operation of the twin-tee combining network linking $D_{9}$ and $D_{10}$.

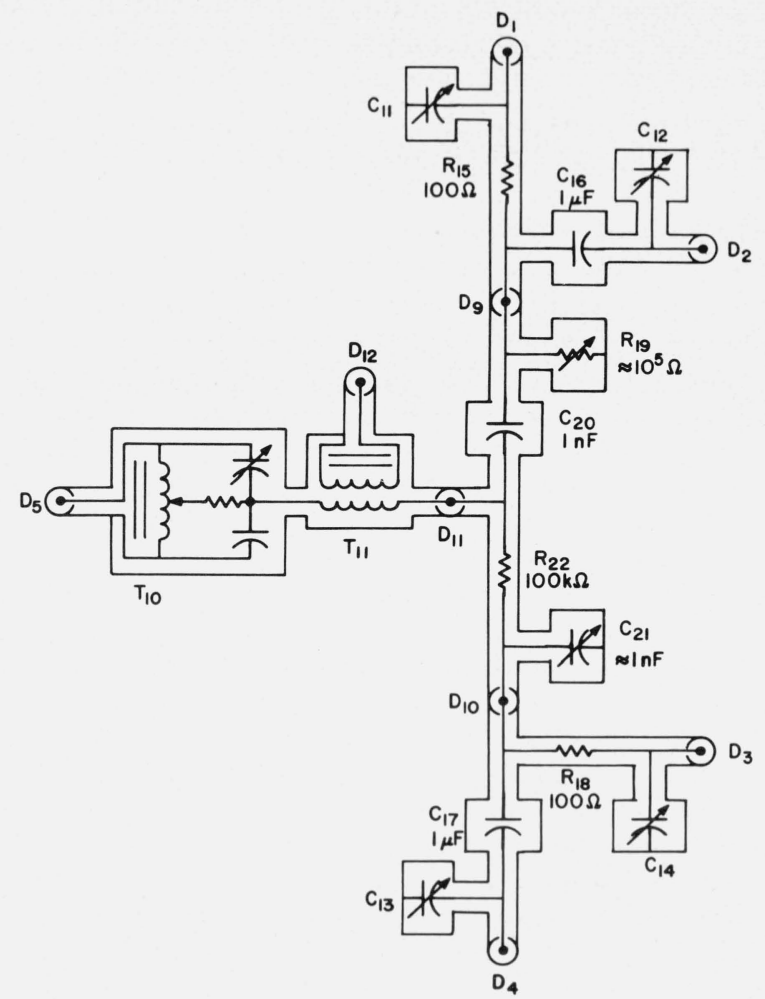

FIGURE 6. Detector combining network for quadrature bridge.

The network linking $D_{9}$ and $D_{10}$ may be understood by letting all generators in figure 5 be zero and by assuming that a generator is inserted to make $e_{c} \neq 0$. The network can be recognized as a twin-tee circuit in which a null can be produced at $D_{11}$ by adjusting $R_{19}$ and $C_{21}$. After making this balance, the voltage at $D_{11}$ is independent of the auxiliary adjustment $Y_{8}+Y_{9}$. In practice the twin-tee is adjusted so that changing $Y_{8}+Y_{9}$ produced no change in the detector voltage at $D_{11}$ and $Y_{8}+Y_{9}$ is adjusted so that changing the twin-tee setting produces no change in the detector voltage at $D_{11}$.

Although a twin-tee combining network is convenient for a quadrature bridge, a detailed noise calculation shows that it rather greatly augments the thermal agitation noise already present in $P_{1}$ and $P_{3}$. With 4 $\mathrm{mW}$ dissipated in $P_{1}$ and $P_{3}$, one has difficulty in detecting an unbalance smaller than one or two parts in $10^{9}$. A substantial improvement could be made by maintaining the combining network at low temperature, or by separately amplifying the voltages at $D_{9}$ and $D_{10}$ before coupling them together.

The final combining network links $D_{11}$ and $D_{5}$ with $D_{12}$. It is adjusted by means of the decade inductive voltage divider $T_{10}$ and the associated phase-shifting network so that inserting a $5-\Omega$ resistor in one of the cables joining $T_{1}$ with $T_{2}, T_{3}$, and $T_{4}$ to simulate the generator shown in figure 5 does not change the detector voltage at $D_{12}$. After making this adjustment the $5-\Omega$ resistor is removed. 
The sequence of adjustments outlined above is easier to perform than to describe. The entire bridge can be adjusted in 10 minutes, and the auxiliary adjustments have been found to drift in a week by less than that required to yield an error of 1 part in $10^{9}$.

The complete quadrature bridge consisting of figures 5 and 6 contains 25 coaxial chokes, each of which was checked using the techniques described in section 2. The largest individual error was found to be 1 part in $10^{10}$, and the total error resulting from the existence of net currents in all chokes, the algebraic sum of the individual errors, was about 2 parts in $10^{11}$.

Some special problems are encountered in a quadrature bridge simply because the balance is frequency-dependent. One especially important problem is caused by intermodulation distortion in the bridge detector between adjacent harmonics of the bridge fundamental frequency. These distortion products may have components at the fundamental frequency, which would cause a substantial error. The individual harmonic components entering the detector of a frequency-dependent bridge are not nulled with the fundamental as they are in a frequency. independent bridge, and may be of rather high amplitude. We use a special filter between $T_{11}$ of figure 6 and the detector amplifier to reject all harmonics of the bridge fundamental frequency before the signals reach any strongly nonlinear elements. The filter contains a bridge network to obtain a zero transfer admittance for the second and third harmonics of the fundamental frequency, and a doubly tuned circuit to attenuate all higher harmonics. The circuit is similar to that used in an earlier quad bridge [7] but has been modified to yield a smaller noise figure.

The need for a good filter is indicated by the fact that removing the filter results in an apparent change in the bridge balance of several parts in $10^{6}$. With the filter in, tests indicate that the error due to intermodulation distortion is less than 1 part in $10^{9}$. A convenient check on the effectiveness of the filter is to augment each of the harmonics present at the detector terminal $D_{12}$ by injecting an additional current into $D_{12}$ with a small capacitor connected to an auxiliary oscillator tuned to the appropriate harmonic. Other tests using two auxiliary oscillators confirm that the effect is indeed due to intermodulation distortion. The technique is very sensitive, and can be used to detect distortion products resulting from nonlinear mixing of frequencies up to at least the 15th harmonic. The technique gives only an upper bound to the error and would probably not be reliable for determining corrections.

If a frequency-dependent bridge is balanced at an angular frequency $\omega=\omega_{0}$, then for $\omega \approx \omega_{0}$, the detector input voltage will be given by $e_{d}=k_{1}\left(\omega-\omega_{0}\right) e_{g}$ where $k_{1}$ is a complex constant and $e_{g}$ is the bridge generator voltage. If the detector contains a sharply tuned single section filter ( $6 \mathrm{~dB} /$ octave $)$ having a bandwidth $2 \omega_{d}$, then for frequencies near $\omega_{0}$ the detector output voltage will be of the form

$$
e_{0}=\frac{k_{2}\left(\omega-\omega_{0}\right) e_{g}}{1+\frac{j\left(\omega-\omega_{0}\right)}{\omega_{d}}} .
$$

The power transfer function between the bridge input terminals and the detector is then given by

$$
T F_{1} \propto \frac{\left(\omega-\omega_{0}\right)^{2}}{1+\left(\frac{\omega-\omega_{0}}{\omega_{d}}\right)^{2}}=\frac{\Delta \omega^{2}}{1+\left(\frac{\Delta \omega}{\omega_{d}}\right)^{2}} .
$$

If a two section filter were used ( $12 \mathrm{~dB} /$ octave), the power transfer function would be

$$
T F_{2} \propto\left(\frac{\Delta \omega}{1+\left(\frac{\Delta \omega}{\omega_{d}}\right)^{2}}\right)^{2} .
$$

$T F_{1}$ and $T F_{2}$ are plotted in figures 7 and 8 for several values of $\omega_{d}$. In both cases, oscillator noise components with frequencies very near $\omega_{0}$ are strongly attenuated, and those at the edge of the filter pass band are the least attenuated.

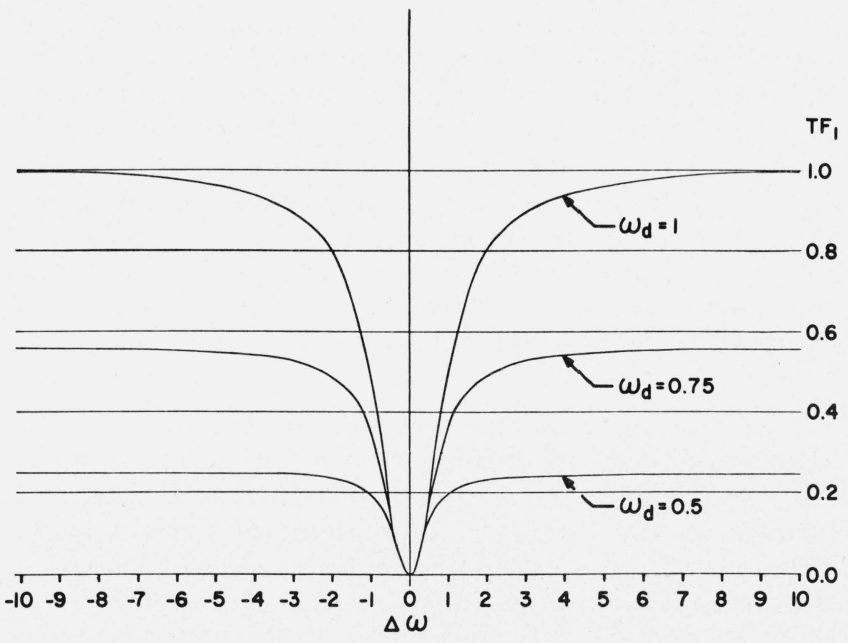

Figure 7. Power transfer functions for a frequency-dependent bridge followed by a single section filter.

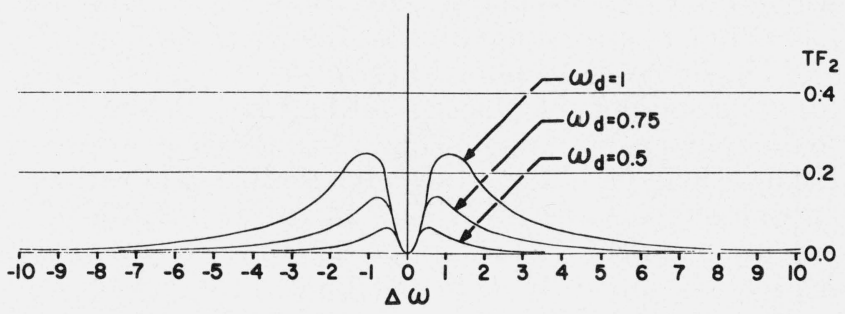

Figure 8. Power transfer functions for a frequency-dependent bridge followed by a double section filter.

The total noise power reaching the detector depends upon the noise spectrum of the oscillator. This is not known a priori, but in general is highest near $\omega_{0}$. We will assume arbitrarily that the noise spectrum is of constant amplitude, in order to simplify the calcula- 
tions. In this case, the total noise power with a single section filter would be of the form

$$
W_{1} \propto \int_{0}^{\infty} \frac{\left(\omega-\omega_{0}\right)^{2}}{1+\left(\frac{\omega-\omega_{0}}{\omega_{d}}\right)^{2}} d \omega \rightarrow \infty
$$

and for a two-section filter,

$$
W_{2} \propto \int_{0}^{\infty}\left[\frac{\omega-\omega_{0}}{1+\left(\frac{\omega-\omega_{0}}{\omega_{d}}\right)^{2}}\right]^{2} d \omega \rightarrow \frac{\pi \omega_{d}^{3}}{2}
$$

The expression (11) for $W_{1}$ is not realistic because (9) is correct only for $\omega \approx \omega_{0}$. In general $T F_{1}$ would decrease when $\Delta \omega$ is very large and would not remain constant, so that $W_{1}$ would be very large but finite.

The advantage of using a two-section filter is obvious from figures 7 and 8 and from (11) and (12), and in addition the very strong dependence of $W_{2}$ on the filter bandwidth can be seen. If the oscillator noise spectrum had a peak at $\omega=\omega_{0}$, the exponent of $\omega_{d}$ in (12) would be reduced, but in practice the observed detector noise due to oscillator noise decreases faster than that due to ordinary thermal agitation noise in the bridge components when the detector bandwidth is decreased. We find with the NBS quadrature bridge connected to a reasonably clean oscillator that the use of two cascaded filters with time constants of $0.3 \mathrm{~s}$ makes the portion of the detector noise due to oscillator noise about equal to that due to thermal agitation noise in the bridge components. Filters with 3 -s time constants are normally employed, in which case the effect of oscillator noise is negligible.

The above discussion deals with noise power and makes no distinction between the different effects of $\mathrm{FM}$ and $\mathrm{AM}$ noise. If a frequency-dependent bridge is connected to a two-phase phase sensitive detector adjusted so that the first channel responds only to the "real" part of the bridge balance and the second channel responds only to the "phase" part of the bridge balance, then the effect of FM noise on the oscillator is to give noise only on the first or real channel of the phase-sensitive detector, as might be expected. It can also be shown that the effect of AM noise on the oscillator is to give noise only on the second or "phase" channel of the detector. The latter effect is not usually anticipated. One consequence of this effect is that when the power level of the bridge is being changed, the phase channel of the detector is thrown off balance, usually by enough to saturate the detector and to require a short wait before the detector recovers. The separation of FM and AM noise with a frequencydependent bridge can be helpful in the study of oscillator noise.

The main components, $P_{1}, P_{2}, P_{3}$, and $P_{4}$ of the NBS quadrature bridge are maintained at $25 \pm 0.001^{\circ} \mathrm{C}$ in an oil bath. The precision of the bridge is limited by the temperature variations in the bath and by the load coefficients of $P_{1}$ and $P_{3}$. The sum of all other errors, including that due to thermal agitation noise, is believed to be less than 1 part in $10^{9}$.

\section{Four-Pair Direct-Reading Ratio Set (DRRS)}

The bridge to be described next was actually the first four-pair admittance bridge to be completed at NBS, and is in some ways not as well conceived as the more recent quadrature bridge described above. It is nevertheless a highly accurate and wide range instrument, and has received much use in the last few years. The bridge has a number of rather complex features, making it difficult to understand without prior experience with four-pair bridges. It is for this reason that its description follows that of the quadrature bridge.

The four-pair direct-reading ratio set was intended to provide a means of comparing two four-pair admittances whose ratio is nominally $10: 1$. It was designed so that it could also be used to compare ordinary two-pair standards. Fewer auxiliary balances are required with the two-pair configuration, so this usage will be described first.

An elementary schematic of the bridge is shown in figure 9. The two admittances to be compared are labeled $Y_{1}$ and $Y_{2}$, and are connected to three star connectors as indicated. The star connectors are constructed in accordance with a design first described by

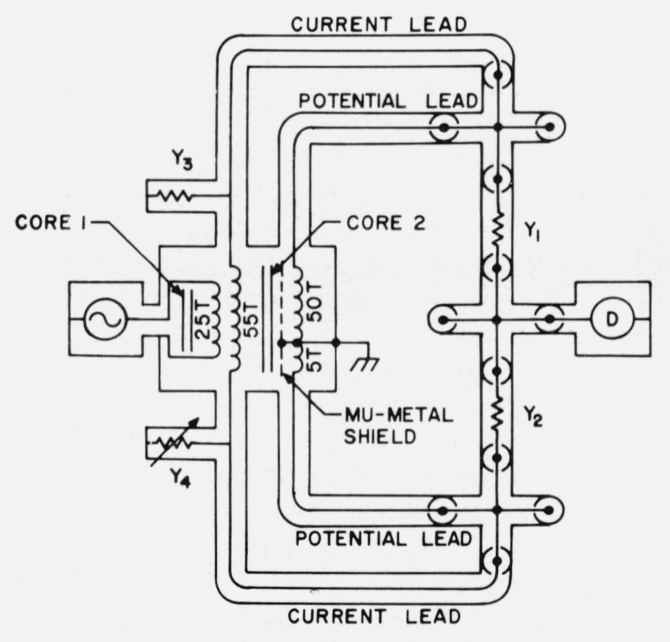

Figure 9. Elementary 10:1 bridge using a two-stage transformer.

Hamon [11] for dc junctions, but are provided with ground potential shields to allow their use with alternating current. The equivalent circuit of an ideal four-pair star connector consists of five impedances linking the five terminals to an inaccessable internal junction point. The impedance to ground is typically much larger than the other four. With this system, the admittances under test are considered to terminate at the internal junction points of the star connectors, rather than at the ends of the coaxial cables leading to the standards. The discrepancy between the two 
definitions of a standard is small if its admittance is small, but could be accounted for to first order.

The transformer in figure 9 is a two-stage device in which the 25-turn primary and the 55-turn current winding are wound around core 1 only. The 50-turn and 5-turn potential windings are wound around both cores and are isolated from the inner windings and the two cores by a mu-metal shield.

If there were no capacitance between windings or to ground, there would be very little current in the potential leads to $Y_{1}$ and $Y_{2}$, and a detector connected to the star connector between $Y_{1}$ and $Y_{2}$ would register a null when $Y_{1} / Y_{2}$ equalled the open circuit ratio of the potential windings. Because of loading effects, both in the potential winding circuit and in $Y_{1}$ and $Y_{2}$ and the associated cables, two auxiliary balances are required to obtain zero current in both potential leads. The first auxiliary balance can be conveniently obtained by means of an adjustable admittance to ground $Y_{4}$ so that $Y_{3}$ and $Y_{4}$ serve as a conventional Wagner circuit, and the second balance can be obtained by means of an auxiliary winding around core 2 only (not shown), whose excitation is adjustable and derived from a high impedance source. It may be noted here that one anticipates that only a very small excitation will be required on core 2 , to drive current through the interwinding capacitance of the transformer potential windings, and that very little voltage will appear across the auxiliary winding on core 2 because of the two-stage construction. Ideally, an adjustable current source could be used to drive core 2 .

The balance procedure for the system of figure 9 would be first to adjust $Y_{1}$ or $Y_{2}$ to achieve a null on the detector; second, to disconnect both potential leads between the transformer and the star connectors and to adjust $Y_{4}$ to restore the null (a balance which is completely independent of the excitation of core 2); and third, to reconnect one of the potential leads and to restore the null by adjusting the core 2 excitation. The process is repeated until the adjustment of $Y_{1} / Y_{2}$ converges to a fixed ratio, which is equal to the open circuit ratio of the transformer potential windings.

A fast convergence of this series of balances is obtained if the equivalent impedences in series with the potential windings are very small with respect to the impedences of the second core excitation circuit and of the Wagner circuit. A technique for eliminating the impedance in series with the high-voltage secondary winding of a three-winding transformer at the expense of slightly increasing the impedence in series with the low-voltage secondary winding has been described [6]. This technique is illustrated by the equivalent circuits based on ideal transformers shown in figures $10 \mathrm{a}$ and $10 \mathrm{~b}$. The circuit of figure $10 \mathrm{a}$ is equivalent to the circuit of figure $10 \mathrm{~b}$, with $z_{1}^{\prime}=z_{1}-9 z_{3}$ and $z_{2}^{\prime}=z_{2}+0.9 z_{3}$ for a transformer with a $10: 1$ ratio. The unlabeled impedences and turns ratio are not precisely equal in the two figures, but this is not important. The significant result is that $z_{3}$ can be adjusted to make $z_{1}^{\prime}=0$.

A technique for making the effective impedance in series with the low potential winding also equal to zero is shown in figure 11. If there is no current in the low potential winding, there will be no voltage across transformer $T_{2}$, and hence no current through the
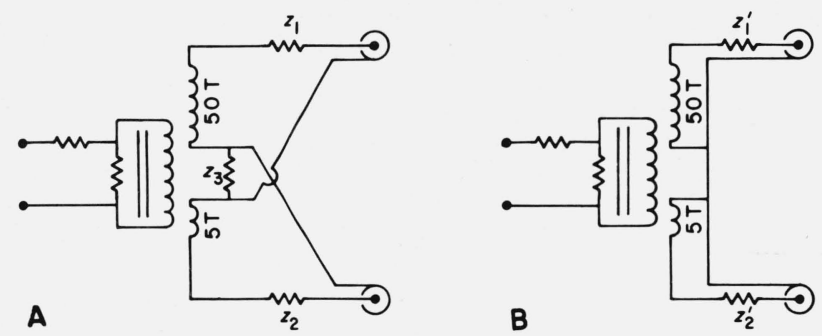

Figure 10. Equivalent circuits for showing the effect of $\mathrm{z}_{3}$ on $\mathrm{z}_{1}^{\prime}$ (see text).

auxiliary admittance $Y_{2}^{\prime}$. However, if there is a current in the potential winding, $T_{2}$ will be excited, producing a compensating current in $Y_{2}^{\prime}$. In the worst case, suppose the potential lead of $Y_{2}^{2}$ is shorted. Then if $z_{2}^{\prime \prime}=z_{2}^{\prime}$, the voltage on the 100 -turn winding of $T_{2}$ will be $e_{1} / 2$, and the voltage applied to $Y_{2}^{\prime}$ will be equal to $e_{1}$. The drastic measure of shorting the potential lead of $Y_{2}$ would then cause no deflection of the null detector provided $Y_{2}^{\prime}=Y_{2}$. An argument based on the circuit linearity indicates that smaller currents in the low potential lead would likewise cause no deflection of the null detector.

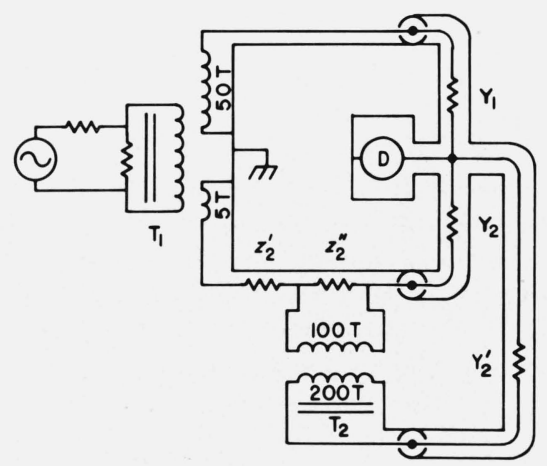

FigURE 11. Compensation scheme for eliminating the effect of $\mathrm{z}_{2}^{\prime}$.

Applying the concepts of figures 10 and 11 to the basic circuit of figure 9 we have a system in which the currents in both potential leads can be adjusted to zero, but in which the bridge balance condition is not strongly dependent upon either auxiliary balance. In order to make the potential lead impedances definite so that $z_{3}$ and $z_{2}^{\prime \prime}$ need only be adjusted once during construction of the bridge, we have elected to plug the star connectors directly into the potential terminals on top of the bridge. The adjustments of $z_{3}$ and $z_{2}^{\prime \prime}$ then serve to make the effective impedances from the internal junction points of the star connectors to the equivalent voltage generators of the transformer potential windings equal to zero, using an auxiliary admittance $Y_{2}^{\prime}=Y_{2}$. It is to be noted that when this 
bridge was designed, the special defining transformers described in section 2 had not yet been invented and the potential leads were open circuited by simply unplugging them. This technique is adequate in this particular case since the potential lead equivalent series impedances are very small. When the junctions between the current and potential leads are located remotely from the bridge, as is the case when measuring four-pair admittances, the lead impedances are not small, and the $\mathrm{W}$ agner and second core excitation adjustments are very critical. The shunt capacitance uncertainties caused by unplugging the coaxial connector could then cause substantial errors.

In order to make a direct reading ratio set out of the circuit of figure 9, a means must be provided for adjusting the ratio of the potential winding in accurately divided steps. The adjustment of the magnitude of the ratio is straightforward, and can be accomplished in principle by means of a step-down transformer $T_{4}$ and a six-decade inductive voltage divider $T_{3}$ magnetically coupled to the potential windings of $T_{1}$ as shown in figure 12. Although $T_{3}$ and $T_{4}$ are represented as being ordinary single-stage transformers, they are actually both two-stage devices. The first stage of $T_{3}$ is coupled to the first stage of $T_{1}$ only, and is used to excite the first stage of $T_{4}$. The switching of the first and second stages of $T_{3}$ are ganged, which improves the accuracy of the voltage division, and results in a negligible interaction with the core 2 excitation adjustment for $T_{1}$.

Constructing an accurate quadrature adjustment for a direct-reading ratio set is much more difficult than constructing an equally accurate magnitude adjustment. At the heart of the problem is the requirement for a $90^{\circ}$ phase-shifting circuit. The conflicting requirements of small output impedance, relatively large output voltage, and reasonably small power dissipation tend to limit the accuracy and range of most passive quadrature balance circuits.

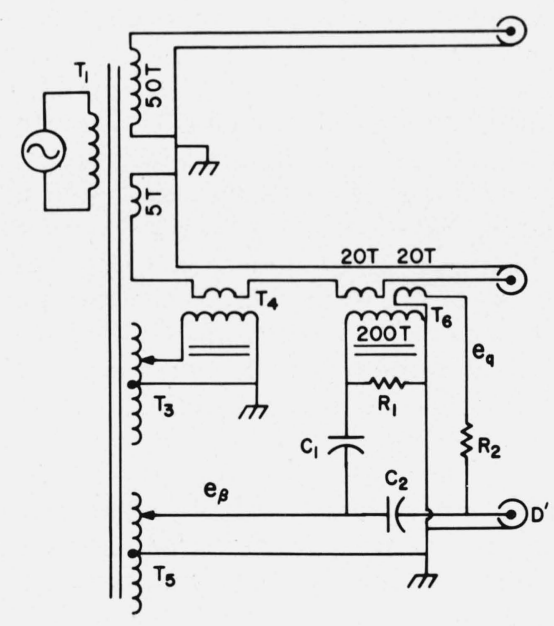

FIGURE 12. Circuitry for adjusting the real and quadrature components of a transformer ratio.
In the quadrature balance system shown in figure 12 , the output of a phase shifting circuit consisting of a $0.01-\mu \mathrm{F}$ capacitor $C_{1}$ and a $10-\Omega$ resistor $R_{1}$ is connected to the primary of the three-winding transformer $T_{6}$. One secondary of $T_{6}$ is in series with the low potential winding of $T_{1} . R_{1}$ is small compared to the input impedance of $T_{6}$, and since $\omega R_{1} C_{1} \ll 1$, the voltage $e_{q}$ added to $T_{1}$ by $T_{6}$ is nearly orthogonal to the voltage across $T_{5}$ and nearly of the desired magnitude. A test of the voltage added to $T_{1}$ by $T_{6}$ can be made by setting up a bridge using a precision $1-n F$ capacitor $C_{2}$ and a precision $10-\Omega$ resistor $R_{2}$. A detector connected to terminal $D^{\prime}$ will register a null when $e_{\beta}$ $j \omega C_{2}=e_{q} / R_{2}$. A small complex adjustment of the excitation to the primary of $T_{6}$ may be made to give a null at $D^{\prime}$, so that $e_{q}=e_{\beta} j \omega C_{2} R_{2}$. Divider $T_{5}$ is a sixdecade, two-stage device similar to $T_{3}$, and in fact has separate windings on ganged switches to separate the coarse output tap connected to $C_{1}$ from the precision output tap connected to $C_{2}$. Resistor $R_{2}$ may at this stage be considered to include the equivalent series impedance of the winding on $T_{6}$ to which it is connected, but in the final design an extra winding was put on $T_{6}$ to provide a four-terminal connection for $R_{2}$.

When the circuits of figures $9,10,11$, and 12 are combined, the result is a system with two sets of detector terminals. The advantages of combining the two to give a single detector whose response is essentially independent of the excitation of $T_{6}$ is by now quite apparent. The NBS bridge has been designed so that connecting an auxiliary admittance $Y_{2}^{\prime \prime}=Y_{2}$ from $D^{\prime}$ to the main null detector $D$ indicated in figure 9 achieves the desired effect. Rather than using two equal auxiliary admittances $Y_{2}^{\prime}$ and $Y_{2}^{\prime \prime}$, a single admittance is used for both purposes by connecting the 200-turn output winding of $T_{2}$ in figure 11 between terminal $D^{\prime}$ in figure 12 and the auxiliary admittance. This serves to add the compensating voltage produced in the quadrature balance circuit of figure 12 to that produced in the load compensation circuit of figure 11.

The complete four-pair direct-reading ratio set contains all of the features described above, and some special compensation circuits for obtaining a quadrature adjustment which is precisely proportional to frequency. The only critical impedances required in the bridge are the capacitor $C_{2}$ and the resistor $R_{2}$ involved in the quadrature adjustment circuit.

The bridge was a test bed for a number of previously untried ideas, and as such it grew in a rather haphazard manner. It is felt that the bridge could be greatly improved if it were rebuilt, and while this is not contemplated at this time, a complete circuit of the existing bridge would be more exposing than revealing, and therefore no such circuit appears in this paper. It is perhaps useful to point out that the key to achieving highly accurate and stable ratios in critical transformers and inductive dividers is to make generous use of multi-stage transformers with magnetic shielding between stages. The principal errors in such transformers are due to capacitive loading effects working on their output impedances. Both the interwinding 
capacitances and the output impedances are minimized by minimizing the number of turns on the transformer. The optimum number of turns on a shielded two-stage transformer designed for audiofrequencies is believed to be less than 100 turns, which is much smaller than the number normally employed in a single-stage design.

The calibration of the bridge was in some ways even more formidable than its construction. The first step was to check the linearity of the bridge dials at several frequencies by an external calibration technique in which a fixed admittance was repeatedly added in parallel with one side of the bridge for various settings of an auxiliary admittance in parallel with the other side. Both magnitude and quadrature balance linearities of all dials were checked in this way. A resolution of 1 part in $10^{10}$ was obtained at $1592 \mathrm{~Hz}$, and of 1 part in $10^{9}$ at all other frequencies.

The second step was to determine the actual bridge settings which would produce an exact 10:1 ratio. A modification of the permutation method described in an earlier paper [12] was used for this purpose.

The last step in the calibration was to determine the actual magnitudes and phase angles corresponding to changes of the real and quadrature dials of the bridge over their entire ranges. A quadrature bridge and a system for comparing admittances in a 1000:1 ratio are needed for this step. Three step-ups using the 10:1 ratio of the bridge under test provided the 1000:1 ratio with ample accuracy.

The bridge accuracy is optimum at $1592 \mathrm{~Hz}$, at which frequency the ratio is adjustable over a range of \pm 5 parts in $10^{4}$ in steps of 1 part in $10^{9}$ for both real and quadrature components. A complete calibration using the procedure outlined above disclosed that the bridge readings are linear at $1592 \mathrm{~Hz}$ over the entire range of the dials within an estimated uncertainty of 3 parts in $10^{10}$. The actual changes in both real and imaginary parts of the bridge ratio produced by changing the dials over their entire ranges differ slightly from the values indicated on the dials. The discrepancies are never greater than 5 parts in $10^{9}$, even when the bridge dials are at the limits of their ranges. Corrections for linear errors of this form are relatively easy to apply.

At $159.2 \mathrm{~Hz}$, the range of the bridge quadrature balance control decreases to \pm 5 parts in $10^{5}$, and at $15920 \mathrm{~Hz}$ it increases to \pm 5 parts in $10^{3}$. The first decade of the quadrature balance control is somewhat nonlinear above $10 \mathrm{kHz}$, but if the bridge is not used for comparing admittances for which the quadrature component of their ratio exceeds \pm 5 parts in $10^{4}$, no errors exceeding 5 parts in $10^{9}$ exist from $159.2 \mathrm{~Hz}$ to $15920 \mathrm{~Hz}$.

The additional circuitry needed when using the bridge for comparing four-pair standards, $P_{1}$ and $P_{22}$, is shown in figure 13. The critical leads to the standards $P_{1}$ and $P_{2}$ are terminated at the reference planes of the defining transformers $T_{7}, T_{8}$, and $T_{9}$, and the leads between these transformers and the standards are treated as part of the standards. The combining network involving $T_{10}$ and the associated phase-shifting network, which joins the detector terminals $D_{1}$ and $D_{2}$ of the two standards, is adjusted to obtain a null on $D_{6}$ when an oscillator is connected to the 100-turn input winding of the defining transformer $T_{8}$. Then the input winding of $T_{8}$ is shorted and the oscillator is reconnected normally. Alternatively, this adjustment can be made by leaving the oscillator connected normally, and adjusting the network so that $D_{6}$ does not respond when the short on $T_{8}$ is removed.

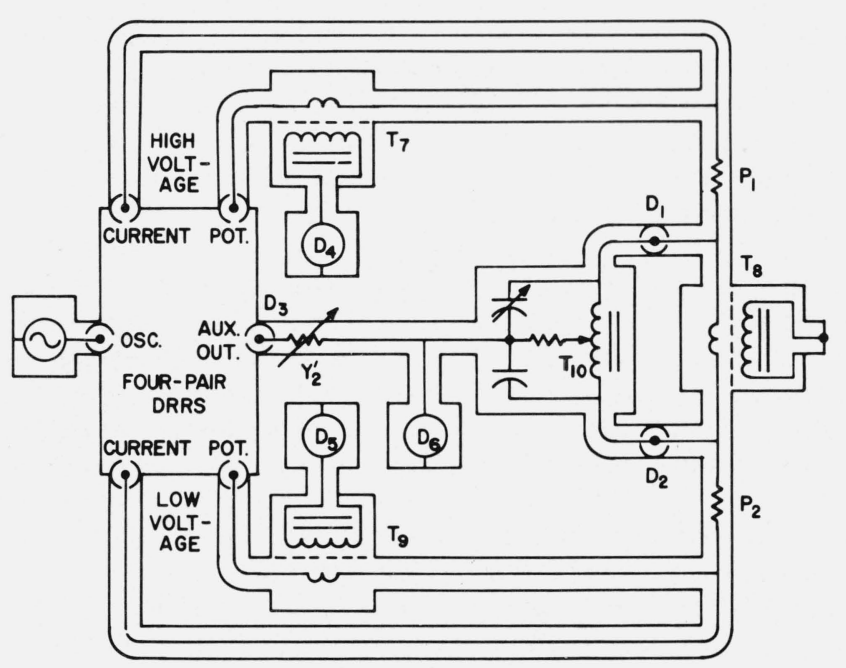

Figure 13. Comparison of four-pair admittances with the four-pair DRRS.

The Wagner balance and second core excitation of the four-pair bridge are adjusted to obtain nulls on detectors connected to points $D_{4}$ and $D_{5}$ of defining transformers $T_{7}$ and $T_{9}$ rather than by disconnecting the potential leads to the bridge. With this system the bridge ratio is defined at the reference planes in $T_{7}$ and $T_{9}$, and differs from the ratio defined at the potential terminals of the bridge. Techniques for comparing the ratios defined in these two ways can be easily developed, or alternatively the permutation method for measuring the bridge ratio [12] can be modified to give the ratio at the reference planes of $T_{7}$ and $T_{9}$ directly. A standard pair of cables for connecting $T_{7}$ and $T_{9}$ to the potential terminals of the bridge is obviously required.

The circuit of figure 13 is not easy to operate directly, partly because the deflections of the auxiliary detectors connected to $D_{4}$ and $D_{5}$ both change when either the bridge Wagner Balance or the second core excitation is changed, and partly because these balances are both much more critical than they are when comparing twopair admittances. The criticalness is caused by the extra equivalent impedances in series with the bridge potential terminals resulting from the added cables between these terminals and the internal junctions of $P_{1}$ and $P_{2}$. A network with the double purpose of combining the output of $D_{5}$ with the main detector and of providing two auxiliary detector outputs, one of which responds to each bridge auxiliary balance, is shown in figure 14.

The 100-turn windings of both $T_{7}$ and $T_{9}$ are terminated with $50-\Omega$ resistors, which when referred to the 
1-turn input windings yields a very small impedance. The voltages across the 100-turn windings are thus proportional to the currents in $T_{7}$ and $T_{9}$. The output voltage at terminal $D_{8}$ is proportional to the sum of these two currents, and responds to changes in the

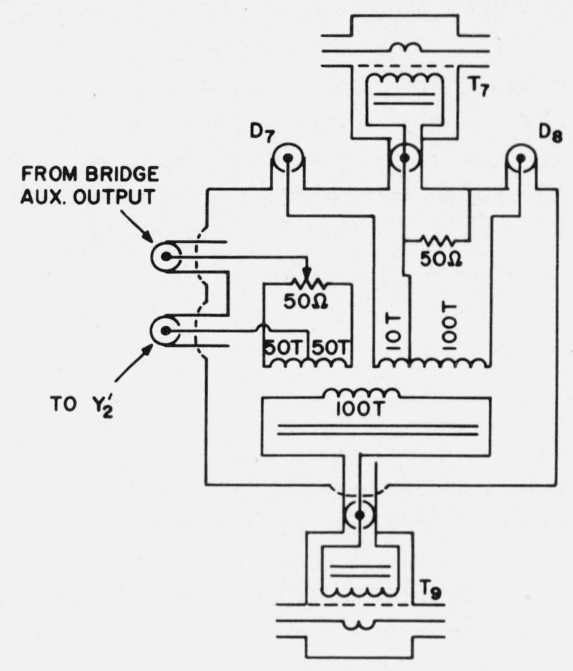

Figure 14. Combining network for assuring fast convergence of DRRS auxiliary balances.

Wagner balance but not to changes in the second core excitation. The output voltage at terminal $D_{7}$ is proportional to the current in $T_{7}$ minus $1 / 10$ of the current in $T_{9}$. It responds to changes in the second core excitation, but not to changes in the Wagner balance. A very rapid convergence of the auxiliary balances can thus be obtained.

A complete combining network for joining the two auxiliary detector points $D_{7}$ and $D_{8}$ with the main detector $D_{6}$ would require two complex adjustments for a total of four adjustable parameters. The simple combining network of figure 14 contains only one adjustable resistor and does not give perfect compensation. It is adequate at $1592 \mathrm{~Hz}$ and below because the reactances of the bridge cables are much smaller than their resistances at this frequency, because the impeddance of the high-voltage potential lead is only onetenth as important as the impedance of the low-voltage potential lead, and because a factor of 10 reduction in the criticalness of the auxiliary balances was found to be sufficient.

A detailed calculation to verify the above conclusions is not appropriate to this paper. The analysis can be carried out with the aid of the equivalent circuit for the bridge potential circuit shown in figure 15, in which $i_{1}$ represents the second core excitation, $i_{2}$ represents the effect of changing the Wagner balance, $i_{3}$ and $i_{4}$ are the currents in the potential leads, $i_{d}$ is the short circuit current at the main detector, and $Y_{1}$ and $Y_{2}$ are the admittances under test. The voltage generator in the current leads of the bridge is represented by $e_{1}$, and can be set equal to zero when analyzing the behavior of the auxiliary balances.

The use of an auxiliary admittance $Y_{2}^{\prime}$ as indicated in figure 13 for combining several null detectors causes no appreciable reduction in bridge sensitivity when the admittances under test are capacitors, but when one is measuring ac resistors this technique substantially increases the thermal agitation noise appearing at the detector. The alternative combining network shown in figure 16 avoids this problem, and works well when the smaller of the resistors under test is $10^{4} \Omega$ or less. The alternative combining network has been

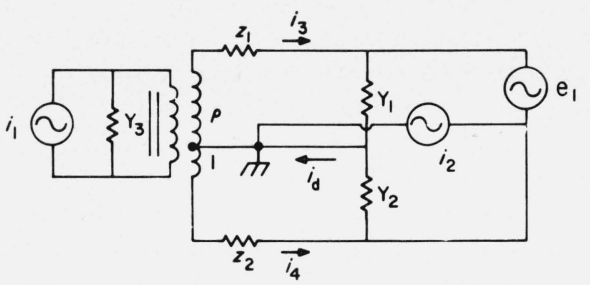

FIGURE 15. Equivalent circuit representing the DRRS auxiliary balances and their effects.

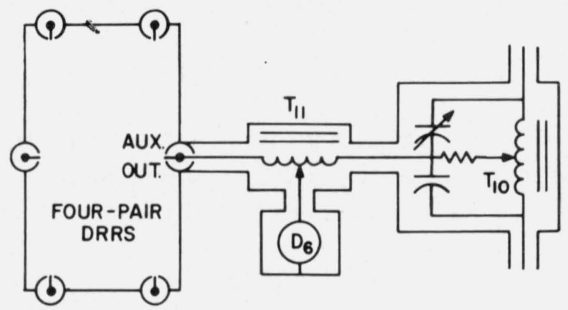

FIGURE 16. Alternative combining network for use when $\left|\mathrm{Y}_{2}\right| \geqslant 10^{-4}$ siemen.

found to be more convenient than the circuit of figure 13 for the measurement of all admittances for which $\left|Y_{2}\right| \geqslant 10^{-4}$ siemens. The circuit of figure 13 is usually easier to adjust when $\left|Y_{2}\right| \leqslant 10^{-5}$ siemens, because it is not as strongly affected by admittances to ground at the junction of $Y_{1}$ and $Y_{2}$ and in the combining network involving $T_{10}$. The circuit of figure 14 works equally well with either combining network.

Although no coaxial chokes are shown in any of the circuits for this bridge, they are obviosuly essential for proper operation. They are inserted where needed to break ground loops, and tested in accordance with the principles laid out in section 2.

\section{Equal-Power 100:1 Resistance Bridge}

The comparison of two resistors whose ratio substantially differs from unity is usually made either by connecting them directly in series and comparing their voltages, or by applying equal voltages to them and comparing their currents. As a result, the powers dissipated in the two resistors differ greatly, and if the load coefficients of the two resistors are about equal, the excitation of the bridge is limited by the load coefficient of the resistor which dissipates the most power. In this case, the bridge sensitivity is much less 
than would be obtained with a unity ratio bridge containing resistors of comparable load coefficients.

Using a voltage transformer bridge such as the directreading ratio set described above, the larger resistor dissipates ten times as much power as the smaller resistor. Using a current transformer bridge such as the conjugate bridge obtained by interchanging generators and null detectors, the smaller resistor dissipates ten times as much power as the larger resistor.

If a bridge contains a voltage transformer of ratio $N_{1}$ and a current transformer of ratio $N_{2}$, then the balance condition is $R_{1}=N_{1} N_{2} R_{2}$; and the powers dissipated in the two resistors are equal if $N_{1}=N_{2}$. The bridge sensitivity in this case is equal to that of a unity ratio bridge for comparing equal resistors. The technique is only applicable for resistors whose ratio is a perfect square, but this is not a fundamental limitation since a rational number can be found that is arbitrarily close to any irrational number.

For the very important case in which the two resistors to be compared have a ratio of 100 , each transformer must have a ratio of 10 . The bridge described in section 4 above contains all of the components required of the voltage transformer in such a system, and in principle another bridge exactly like it but with a subtractive rather than an additive ratio could be used for the current transformer. Fortunately the current transformer part of the bridge need not be provided with an adjustable ratio, and is therefore much easier to build. A quite different approach to an equal-power, four-terminal (but not four-pair) impedance bridge has been developed by Henry Hall of the General Radio Company.2 Both two-pair and four-pair equal-power bridges have been described in the literature [12], but the operation of the four-pair version is not clear unless two-stage transformers are employed.

A paper describing this work was presented at the 1970 Conference on Precision Electromagnetic Measurements at Boulder, Colorado. The text of this paper will be published in an issue of the IEEE Transactions on Instrumentation and Measurement.
The 100:1 bridge used at NBS for comparing a $10^{3}-\Omega$ four-pair resistor with a $10^{5}-\Omega$ four-pair resistor at $1592 \mathrm{~Hz}$ is shown in figure 17. It is convenient for the purpose of analyzing the behavior of this circuit mentally to interchange all generators and detectors and to work with the conjugate bridge. Although the circuit could of course be described directly without making use of the fictitious interchange, and although this would seem a more natural approach to people familiar with current comparators, the interchange is helpful here because it allows the use of the same terminology as was developed for describing the direct reading ratio set of section 4 .

If we consider then that a generator is connected to terminal $B$ in figure 17 , it can be seen that the second core excitation circuitry is identical with that described in section 4 , but that the Wagner circuitry is much more complex. The Wagner circuit combines a 10:1 voltage transformer $T_{15}$ with a 10:1 current transformer $T_{16}$ to obtain simultaneously nearly the correct 10:1 voltage ratio and 10:1 current ratio in the two resistors under test. Note that the ratio of currents in the two windings of $T_{16}$ is nominally equal to the turns ratio, so that very little voltage appears across either winding. The two auxiliary adjustments then provide only the small corrections necessary to meet exactly the required conditions of zero current in the defining transformers $T_{12}$ and $T_{13}$.

Although a much simpler Wagner balance system involving resistors could have been used, the circuit chosen is superior because it leads to a negligible augmentation of the thermal agitation noise appearing at a detector connected at point $B$. This consideration is of no importance in the Wagner circuit of the voltage transformer part of the bridge, and the use of $R_{3}$ to extend the limited range of the internal Wagner adjustment of the direct-reading ratio set is perfectly satisfactory.

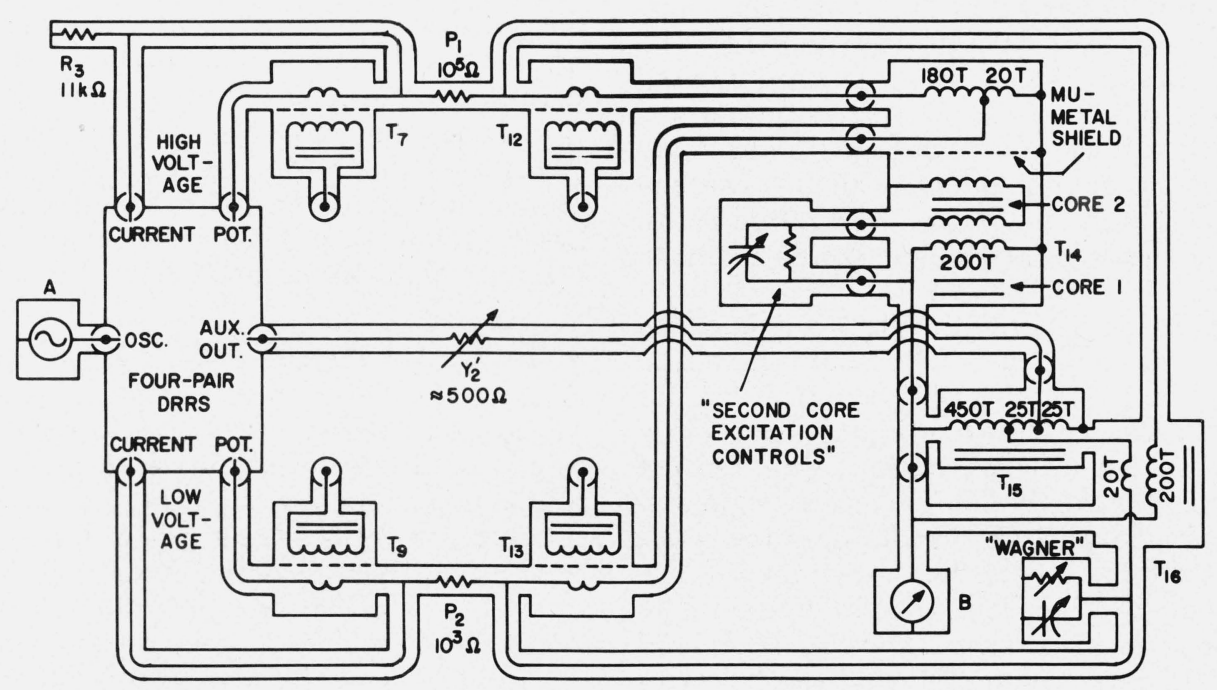

FIGURE 17. A 100:1 equal power bridge making use of the four-pair DRRS. 
The auxiliary resistor required for proper operation of the quadrature balance controls of the direct-reading ratio set would most conveniently consist of a $10^{4}-\Omega$ resistor connected between the auxiliary output terminal of the bridge and the detector point $B$. This would produce an unacceptably large thermal agitation noise. The use of a $500-\Omega$ auxiliary resistor for $Y_{2}^{\prime}$ which is connected to a tap on $T_{15}$ eliminates this problem. This does not interfere with the use of the circuit of figure 14 to reduce the criticalness of the direct-reading ratio set auxiliary balances.

A circuit similar to that of figure 14 and connected to defining transformers $T_{12}$ and $T_{13}$ is used to reduce the criticalness of the current transformer auxiliary balances and to provide a means of quickly making the current transformer auxiliary balances. This circuit is shown in figure 18. An auxiliary admittance $Y_{3}$ (not shown) is connected between this circuit and the generator terminal $A$ on the direct-reading ratio set.

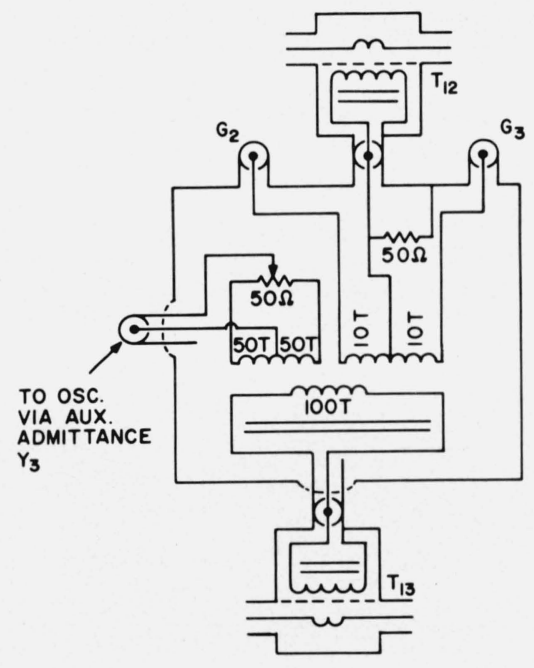

FiguRE 18. Combining network for assuring fast convergence of current transformer auxiliary balances.

The circuit of figure 18 is complimentary to the circuit of figure 14. In use, the current transformer auxiliary balances are adjusted so that temporarily connecting a generator to either $G_{2}$ or $G_{3}$ produces no change in the detector connected to terminal $B$ in figure 17. The adjustable $50-\Omega$ resistor in figure 18 or the auxiliary admittance $Y_{3}$ is set so that these auxiliary balances are not critical.

It was necessary to compromise in choosing the number of turns for transformer $T_{14}$ in figure 17 in order to achieve a reasonably stable ratio without unduly augmenting the thermal agitation noise due to excessive shunt conductance in the transformer. With 200 turns on $T_{14}$, the shunt conductance measured at point $B$ with $P_{1}$ and $P_{2}$ removed was found to be $1.8 \times 10^{-5}$ siemens which is only slightly less than the $2 \times 10^{-5}$ siemens conductance contributed by the resistors under test. With $4 \times 10^{-3} \mathrm{~W}$ dissipated in each resistor $\left(20 \mathrm{~V}\right.$ on $\left.P_{1}\right)$, the sensitivity is sufficient to resolve 1 part in $10^{9}$ using a phase-sensitive detector followed by a recorder, which is considered to be adequate.

The easiest way to measure the ratio of the current transformer $T_{14}$ is to interchange the connections with respect to the direct-reading ratio set so that a balance is obtained when $P_{1}$ is nominally equal to $P_{2}$. Interchanging $P_{1}$ and $P_{2}$ and rebalancing allows the current transformer ratio to be determined from the known ratio of the direct-reading ratio set. A slight change in the Wagner circuit of the current transformer is also required. This consists of interchanging the windings of $T_{16}$. Resistors cannot be used for $P_{1}$ and $P_{2}$ because of their large load coefficients, so a pair of $1-n F$ capacitors is used instead. The voltages on the two capacitors change from $20 \mathrm{~V}$ to $200 \mathrm{~V}$ in the course of the measurement, so their voltage dependencies must be accurately known. Techniques for measuring the voltage dependencies of capacitors to the required accuracy have been described by Shields [14].

\section{Conclusions}

The series of bridges described above were built to provide the admittance comparisons necessary for an absolute ohm determination, as sketched in the introduction. It has been found that all of the comparisons necessary for this work can be made with an overall uncertainty of a few parts in $10^{9}$ using these bridges. In practice, the largest source of uncertainty results from instabilities in the admittance standards used in the measurements. This problem can be handled only be carefully controlling the temperature of the standards.

\section{References}

[1] Cutkosky, R. D., Four-terminal-pair networks as precision admittance and impedance standards, commun. and Electronics 70, 19-22 (Jan. 1964).

[2] Homan, D. N., Some techniques for measuring small mutual inductances, J. Res. Nat. Bur. Stand. (U.S.), 70C (Eng. and Instr.), No. 4, 221-226 (Oct.-Dec. 1966).

[3] Haddad, R. J., A resistor calculable from DC to $\omega=10^{5} \mathrm{rad} / \mathrm{s}$, Master's thesis, School of Engineering and Applied Science, The George Washington University, Washington, D.C., 1-57 (Apr. 1969).

[4] Thompson, A. M., AC bridge methods for the measurement of three-terminal admittances, IEEE Trans. Instr. Meas. IM-13, No. 4, 189-197 (Dec. 1964).

[5] Kavanagh, R. J., Noninteracting controls in linear systems, Trans. AIEE 76, part 2, 95-100 (May 1957).

[6] Cutkosky, R. D., Active and passive direct reading ratio sets for the comparison of audio-frequency admittances, J. Res. Nat. Bur. Stand. (U.S.), 68C (Eng. and Instr.), No. 4, 227-236 (Oct.-Dec. 1964).

[7] Cutkosky, R. D., Evaluation of the NBS unit of resistance based on a computable capacitor, J. Res. Nat. Bur. Stand. (U.S.), 65A (Phys. and Chem.), No. 3, 147-158 (May-June 1961).

[8] Thompson, A. M., An absolute determination of resistance based on a calculable standard of capacitance, Metrologia 4, No. 1, 1-7 (Jan. 1968).

[9] Millea, A., and Ilie, P., A class of double-balance quadrature bridges for the intercomparison of three-terminal resistance, inductance and capacitance standards, Metrologia 5, No. 1, 14-20 (Jan. 1969).

[10] Brooks, H. B., and Holtz, F. C., The two-stage current transformer, AIEE Trans. 41, 382-393 (1922). 
[11] Hamon, B. V., A 1-100 $\Omega$ build-up resistor for the calibration of standard resistors, J. Sci. Instr. 31, 450-453 (1954).

[12] Cutkosky, R. D., and Shields, J. Q., The precision measurement of transformer ratios, IRE Trans. Instr. I-9, No. 2, 243-250 (1960).

[13] Clark, H. A. M., and Vanderlyn, P. B., Double-ratio AC bridges with inductively-coupled ratio arms, Proc. IEE 96, Pt. 3, 189-202 (1949).
[14] Shields, J. Q., Voltage dependence of precision air capacitors, J. Res. Nat. Bur. Stand. (U.S.), 69C (Eng. and Instr.), No. 4, 265-274 (Oct.-Dec. 1965).

(Paper No. 74C3\&4-299) 